\title{
All-optical pump-and-probe detection of two-time correlations in a Fermi gas
}

\author{
T.-L Dao, ${ }^{1,2}$ C. Kollath, ${ }^{1}$ I. Carusotto,${ }^{3}$ and M. Köhl ${ }^{4}$ \\ ${ }^{1}$ Centre de Physique Théorique, École Polytechnique, CNRS, 91128 Palaiseau, France. \\ ${ }^{2}$ Laboratoire Charles Fabry de l'Institut d'Optique, CNRS and Univ. Paris-Sud, \\ Campus Polytechnique, RD 128, F-91127 Palaiseau cedex, France. \\ ${ }^{3}$ CNR-INFM BEC Center and Dipartimento di Fisica, Università di Trento, 38050 Povo, Italy \\ ${ }^{4}$ Cavendish Laboratory, University of Cambridge, JJ Thomson Avenue, Cambridge CB3 OHE, United Kingdom
}

(Dated: October 31, 2018)

\begin{abstract}
We propose an all-optical scheme to probe the dynamical correlations of a strongly-interacting gas of ultracold atoms in an optical lattice potential. The proposed technique is based on a pump-and-probe scheme: a coherent light pulse is initially converted into an atomic coherence and later retrieved after a variable storage time. The efficiency of the proposed method to measure the two-time one-particle Green function of the gas is validated by numerical and analytical calculations of the expected signal for the two cases of a normal Fermi gas and a BCS superfluid state. Protocols to extract the superfluid gap and the full quasi-particle dispersions are discussed.
\end{abstract}

PACS numbers: 03.75.Ss, 42.50.Gy, 78.47.jc, 71.10.Fd

\section{INTRODUCTION}

Many-body quantum systems exhibit truly remarkable features such as high-temperature superconductivity and the fractional quantum Hall effect. Traditionally, these phenomena are studied in the solid state. However, in recent years dilute, yet strongly interacting, atomic gases have started providing a novel class of systems to investigate this fascinating physics. Their outstanding cleanliness, control, and precise microscopic understanding will push forward the fundamental understanding of quantum many-body physics [1].

Strongly interacting atomic quantum gases are generally prepared by trapping atoms in vacuum in a magnetic or optical potential. This offers two remarkable opportunities: Firstly, a superb isolation from the environment opens the door to fascinating experiments out of equilibrium to investigate genuine quantum dynamics. Secondly, a variety of coherent optical processes are available to selectively probe the quantum system without being disturbed by a surrounding bulk medium. In particular, these optical detection techniques can provide repetitive and almost non-destructive in-situ measurements [2, 3]. The combination of these two features makes ultracold quantum gases ideal systems to study the non-equilibrium and dynamic properties of isolated quantum many body systems. However, the experimental study of these properties requires the development of novel detection schemes that are sensitive to a wider variety of observables of the quantum gas, e.g. its multi-time correlation functions.

The prime example of an atomic quantum system mimicking the physics of the solid state are interacting fermionic atoms in artificial lattices structures, the so called optical lattices [4]. The preparation of strongly correlated states in an optical lattice will allow for an analog simulation of complex quantum many body Hamiltonians. Recently, evidence for the stabilization of a Mott-insulating phase has been obtained by looking at density related quantities of the gas $[5-8]$. The identification and characterization of more complex quantum phases requires, however, the measurement of time-resolved single-particle correlation functions, also called Green func- tions, of the form $\left\langle\psi_{\sigma, r}^{\dagger}(t) \psi_{\sigma^{\prime}, r^{\prime}}\left(t^{\prime}\right)\right\rangle$. Here $\psi_{\sigma, r}^{(\dagger)}(t)$ is the annihilation (creation) operator for the internal atomic state $\sigma$ at position $r$ and time $t$. The single-particle two-time correlation function reveals profound information about the macroscopic coherence and decoherence of the systems and keeps track of the subtle properties of quantum phases which are not densityordered, e.g. the existence of quasi-particles in a strongly correlated Fermi liquid. This same correlation function plays a even more crucial role in the case non-equilibrium situations: as the most celebrated example, the particular relaxation behaviour of glasses is almost invisible in one-time correlations, while it can be followed in full detail by measuring the twotime ones [9].

Up to now, the single particle equal time correlation function out of equilibrium was investigated for bosonic atoms [10]. For fermions, only the energy resolved correlation function of an equilibrium state has so far been probed by momentum-integrated [11-16] and momentum resolved [17, 18] radio-frequency or two-photon spectroscopy. A more elaborated scheme for the detection of the two-time correlation function based on the immersion of a ion into quantum gases has been proposed in [19].

Here, we propose an all-optical pump-and-probe scheme to extract quantitative information on the microscopic physics of a Fermi gas and in particular on its two-time correlation functions. A pump sequence firstly brings the system into a quantum superposition of its initial state and an excited state. The response of the system to a second probe pulse sequence is then measured after a variable time delay. In this way, information on the time evolution of the atomic two-time correlations is converted into easily detectable observables, such as the intensity and the phase of the outgoing light.

From an alternative point of view, our scheme can be seen as an application of light storage techniques [20-22] to the diagnostic of many-body systems: a coherent pulse of light is stored in a quantum gas and retrieved at a later time after a variable interval. Information on the system is extracted from the properties of the retrieved light. Differently from standard light storage experiments where it is a purely detrimental effect, decoherence of the stored pulse as a function of storage 
time is in our scheme the crucial tool to obtain information on the many-body dynamics of the underlying quantum gas.

The structure of the paper is the following. In Sec III the measurement schemes are introduced and analytical expression relating the observed signal to the many-body observables are given. In Sec $\amalg$ an application to a fermionic system is discussed in detail and experimental protocols to extract the superfluid gap and the full quasi-particle dispersions of a BCS superfluid are outlined. Conclusions and future perspectives are given in SecIV

\section{THE MEASUREMENT PROCEDURE}

(a)

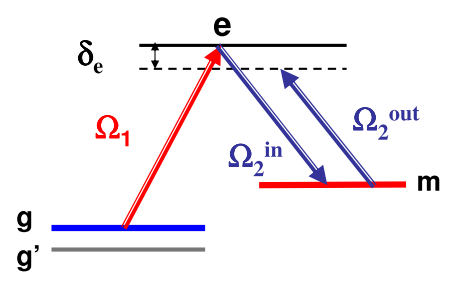

FIG. 1: (Color online) Diagram of the internal atomic levels involved in the proposed detection scheme.

(b)

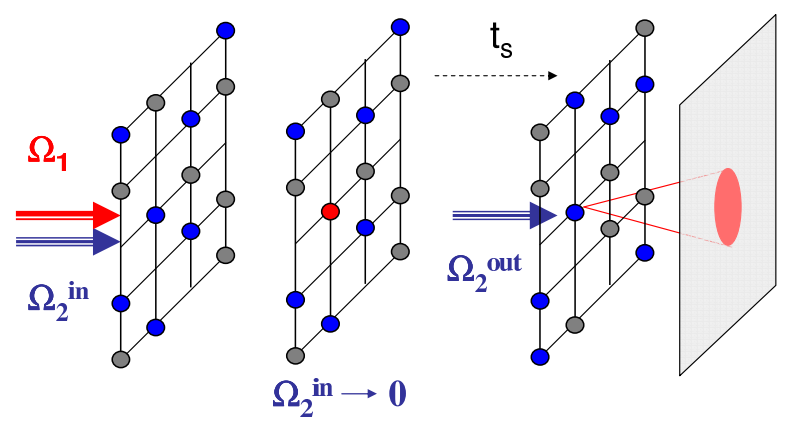

FIG. 2: (Color online) Snapshots of the measurement procedure. From left to right: adiabatic storage of coherence from incident beams, free many-body evolution, light re-emission and detection.

Even though the application scope of our measurement procedure is much wider, we focus our attention here onto the case of fermionic atoms in a two-dimensional lattice geometry. This geometry lies at the heart of quantum simulations with the aim of exploring the mechanisms underlying high-temperature superconductivity [23]. A tight optical confinement potential freezes the atomic motion into a single $x y$ plane. Additionally, a periodic optical lattice potential is applied along the $x$ and $y$ directions to generate a twodimensional lattice structure [24].
The gas consists of a mixture of atoms in two hyperfine ground states $g$ and $g^{\prime}$ that feel an identical confinement potential. Our all-optical probing scheme involves three atomic levels $(g, e$, and $m)$ arranged in a $\Lambda$ scheme as schematically shown in Fig.11 The $m$ state is a long-lived electronic ground state whereas the $e$ state is an electronically excited state. With a suitable choice of polarization and frequency, the $g^{\prime}$ atoms experience a negligible coupling to the pump and probe light fields.

The diagnostic scheme (Fig. 22) starts with the creation of a coherent excitation by adiabatically switching on a laser of (spatially uniform) Rabi frequency $\Omega_{2}^{\text {in }}$ and then a weaker collinear laser of (spatially dependent) Rabi frequency $\Omega_{1}(\mathbf{r})$ [25]. The two beams are then suddenly and simultaneously switched-off. The frequency $\omega_{1,2}$ of the beams are chosen to be resonant with the $g \rightarrow e$ and $m \rightarrow e$ transitions, respectively. To ensure adiabaticity of the preparation stage, the switch-on of the two lasers has to be performed on a timescale long as compared to the internal atomic dynamics and to the Rabi frequencies $\Omega_{1}, \Omega_{2}^{\text {in }}$. On the other hand, the switchoff has to be much faster than all these frequencies.

Provided the whole excitation stage takes place on a fast time scale as compared to the many-body dynamics, the atomic position can be considered as fixed and the optical process described by a single atom picture where the atomic $|g\rangle$ state is adiabatically transformed into a dark state [26-29]

$$
\mid \text { dark }\rangle=\frac{e^{i \theta}}{\sqrt{\left|\Omega_{1}\right|^{2}+\left|\Omega_{2}^{i n}\right|^{2}}}\left[\Omega_{2}^{i n}|g\rangle-\Omega_{1}|m\rangle\right]
$$

which is fully decoupled from the excitation lasers. All other bright eigenstates are energetically separated and do not get mixed with the dark state provided the switch-on phase is performed in a slow enough manner. Assuming that the phase of the $\Omega_{1,2}$ Rabi frequencies (once the carriers at $\omega_{1,2}$ are eliminated) is constant during the whole sequence, the global Berry phase

$$
\theta=i \int d t\left\langle\operatorname{dark}(t)\left|\frac{d}{d t}\right| \operatorname{dark}(t)\right\rangle
$$

acquired by the atom during the adiabatic evolution is easily shown to vanish.

In the $\left|\Omega_{1}\right| \ll\left|\Omega_{2}^{\text {in }}\right|$ limit under investigation here, the effect of the excitation stage on the initial many-body state $\left|\phi_{0}\right\rangle$ in the lattice representation can be expressed as the transformation:

$$
\left|\phi_{d}\right\rangle \simeq\left(\mathbf{1}-\sum_{i} \frac{\Omega_{1}\left(\mathbf{r}_{i}\right)}{\Omega_{2}^{\text {in }}} \hat{\psi}_{m, \mathbf{r}_{i}}^{\dagger} \hat{\psi}_{g, \mathbf{r}_{i}}\right)\left|\phi_{0}\right\rangle .
$$

Here, the $\hat{\psi}_{\sigma, \mathbf{r}_{i}}\left(\hat{\psi}_{\sigma, \mathbf{r}_{i}}^{\dagger}\right)$ lattice operators destroy (create) a fermionic atom in the $\sigma=g, m$ state at the lattice site $\mathbf{r}_{i}$, respectively. $\Omega_{1,2}$ are the Rabi frequencies in the lattice representation. Initially, only the $g$ and $g^{\prime}$ states are assumed to be occupied.

The use of a locally focused laser of amplitude $\Omega_{1}(\mathbf{r})$ on the $g \rightarrow e$ transition allows one to selectively address a well defined region of the sample. The space selection is useful to 
eliminate inhomogeneous broadening effects due to the spatially varying density in e.g. trapped systems [30, 31]. If no spatial selection is performed, the final signal would include the contributions of different regions of the system.

Once the two beams are switched off, the system evolves according to its many-body Hamiltonian for a storage time $t_{s}$ from the state $\left|\phi_{d}\right\rangle$ to the new many-body state

$$
\left|\phi\left(t_{s}\right)\right\rangle=U_{m b}\left(t_{s}\right)\left|\phi_{d}\right\rangle=e^{-i\left(H_{0}+H_{m}+H_{\mathrm{int}}\right) t_{s} / \hbar}\left|\phi_{d}\right\rangle
$$

where $U_{m b}\left(t_{s}\right)$ is the many body time-evolution operator and the system Hamiltonian involves three contributions: $H_{0}$ is the Hamiltonian acting on the states $g$ and $g^{\prime}, H_{m}$ the Hamiltonian of the atoms in the $m$ state, and $H_{\text {int }}$ contains the interaction processes between the $g, g^{\prime}$ and $m$ states. This timeevolution will change the coherence between $g$ and $m$ present in the prepared dark state. The $g, m$ coherence remaining at the end of the storage time is finally probed.

The detection of the remaining coherence can be achieved by different schemes: Either a fast $\pi$ pulse is applied to coherently transfer all atoms from the $m$ to the $e$ state and then coherent photons emitted on the $e \rightarrow g$ transition are detected. Or the excitation is slowly released by means of a weak field of frequency $\omega_{2}$ and Rabi frequency in the continuum $\Omega_{2}^{\text {out }} \ll \gamma_{e}$ that transfers the atoms adiabatically from the $m$ state into a coherent superposition of $m$ and $e$. In both cases, the electric dipole that is responsible for the emission at frequency $\omega_{1}$ on the $e \rightarrow g$ transition is proportional to the coherence between the $g$ and $m$ atomic states,

$$
\hat{d}(\mathbf{r})=D \hat{\psi}_{g, \mathbf{r}}^{\dagger} \hat{\psi}_{m, \mathbf{r}} .
$$

The constant $D$ depends on the details of the process and determines the duration in time $\tau_{r}$ of the released pulse $\tau_{r}^{-1}=$ $\gamma_{e}\left|d_{e g} / D\right|^{2}$, where $\gamma_{e}$ is the radiative decay rate of $e$ state atoms. In the $\pi$ pulse case, the constant $D$ is equal to the electric dipole matrix element between the state $g$ and $e, D=d_{g e}$. In the case of a slow release, $D$ is approximately given by $D=2 i \Omega_{2}^{\text {out }} d_{g e} / \gamma_{e}$. In order for the many-body dynamics not to interfere with the release process, the time duration $\tau_{r}$ of this latter has to be shorter than the characteristic time scales of the many-body dynamics.

The near field pattern of the emitted light amplitude is determined by the expectation value of the local dipole operator on the final state $\left|\phi\left(t_{s}\right)\right\rangle$

$$
d\left(\mathbf{r}, t_{s}\right) \quad=\left\langle\phi\left(t_{s}\right)|\hat{d}(\mathbf{r})| \phi\left(t_{s}\right)\right\rangle
$$

We switch now to the lattice representation by relating the field operators in the continuum to the lattice operators via the Wannier functions $w_{\sigma}(\mathbf{r})$ as following $\hat{\psi}_{\sigma, \mathbf{r}}=\sum_{i} w_{\sigma}(\mathbf{r}-$ $\left.\mathbf{r}_{i}\right) \hat{\psi}_{\sigma, \mathbf{r}_{i}}$. We checked numerically that for tight atomic Wannier functions on deep lattices, we can keep only the terms with Wannier factors taken at the same sites and neglect all other contributions. This leads to the following expression for the dipole operator

$$
d\left(\mathbf{r}, t_{s}\right) \simeq D \sum_{i} W_{\mathbf{r}_{i}}(\mathbf{r})\left\langle\phi\left(t_{s}\right)\left|\hat{\psi}_{g, \mathbf{r}_{i}}^{\dagger} \hat{\psi}_{m, \mathbf{r}_{i}}\right| \phi\left(t_{s}\right)\right\rangle,
$$

with $W_{\mathbf{r}_{i}}(\mathbf{r})=w_{g}^{*}\left(\mathbf{r}-\mathbf{r}_{i}\right) w_{m}\left(\mathbf{r}-\mathbf{r}_{i}\right)$. Inserting the expression (4) of the final state and switching to the Heisenberg representation for the operators $\hat{\psi}_{x, \mathbf{r}_{i}}\left(t_{s}\right)=U_{m b}^{\dagger}\left(t_{s}\right) \hat{\psi}_{x, \mathbf{r}_{i}} U_{m b}\left(t_{s}\right)$, this has the form

$$
\begin{aligned}
d\left(\mathbf{r}, t_{s}\right)= & D \sum_{i} W_{\mathbf{r}_{i}}(\mathbf{r}) \\
& \times\left\langle\phi_{d}\left|U_{m b}^{\dagger}\left(t_{s}\right) \hat{\psi}_{g, \mathbf{r}_{i}}^{\dagger} \hat{\psi}_{m, \mathbf{r}_{i}} U_{m b}\left(t_{s}\right)\right| \phi_{d}\right\rangle \\
=D & \sum_{i} W_{\mathbf{r}_{i}}(\mathbf{r})\left\langle\phi_{d}\left|\hat{\psi}_{g, \mathbf{r}_{i}}^{\dagger}\left(t_{s}\right) \hat{\psi}_{m, \mathbf{r}_{i}}\left(t_{s}\right)\right| \phi_{d}\right\rangle .
\end{aligned}
$$

Inserting into (8) the explicit expression (3) for the dark state and taking into account that no atoms were initially present in the $m$ state, this expression can be written in the compact form

$$
\begin{aligned}
& d\left(\mathbf{r}, t_{s}\right)=-\frac{D}{\Omega_{2}^{\text {in }}} \sum_{i, j} \Omega_{1}\left(\mathbf{r}_{j}\right) W_{\mathbf{r}_{i}}(\mathbf{r}) \\
& \quad \times\left\langle\phi_{0}\left|\hat{\psi}_{g, \mathbf{r}_{i}}^{\dagger}\left(t_{s}\right) \hat{\psi}_{m, \mathbf{r}_{i}}\left(t_{s}\right) \hat{\psi}_{m, \mathbf{r}_{j}}^{\dagger}(0) \hat{\psi}_{g, \mathbf{r}_{j}}(0)\right| \phi_{0}\right\rangle
\end{aligned}
$$

that only involves a time-dependent correlation function taken on the initial many-body state $\left|\phi_{0}\right\rangle$.

As no atoms are initially present in the $m$ state, the initial many-body state $\left|\phi_{0}\right\rangle$ exactly factorizes in a complex manybody state for the $g, g^{\prime}$ subspace and vacuum for the $m$ one. Assuming that the few atoms that are transferred into the $m$ state during the preparation stage do not significantly interact with the majority of atoms left in the $g, g^{\prime}$ states [18, 32, 33] allows us to neglect the $m-g, g^{\prime}$ interaction term of the Hamiltonian $H_{\text {int }}$ in (4) and write the time-evolution operator $U_{m b}$ in the factorized form $U_{m b}\left(t_{s}\right)=e^{-i H_{0} t_{s} / \hbar} e^{-i H_{m} t_{s} / \hbar}$. As a direct consequence, the Heisenberg evolution of the $\hat{\psi}_{m, \mathbf{r}_{i}}(t)$ operator is only determined by the $H_{m}$ part of the evolution operator, while the $\hat{\psi}_{g, \mathbf{r}_{i}}(t)$ and $\hat{\psi}_{g^{\prime}, \mathbf{r}_{i}}(t)$ operators evolve with the many-body $H_{0}$ Hamiltonian in the $g, g^{\prime}$ space. These simple facts allow one to rewrite the dipole expectation value in the final form:

$$
\begin{aligned}
d\left(\mathbf{r}, t_{s}\right)= & \left.-\frac{D}{\Omega_{2}^{\text {in }}} \sum_{i, j} \Omega_{1}\left(\mathbf{r}_{j}\right)\right) W_{\mathbf{r}_{i}}(\mathbf{r}) \\
& \times\left\langle\operatorname{vac}\left|\hat{\psi}_{m, \mathbf{r}_{i}}\left(t_{s}\right) \hat{\psi}_{m, \mathbf{r}_{j}}^{\dagger}(0)\right| \operatorname{vac}\right\rangle \\
& \times\left\langle\phi_{0}\left|\hat{\psi}_{g, \mathbf{r}_{i}}^{\dagger}\left(t_{s}\right) \hat{\psi}_{g, \mathbf{r}_{j}}(0)\right| \phi_{0}\right\rangle .
\end{aligned}
$$

where all the expectation values are to be evaluated on the initial many-body state before the preparation stage, with no occupation in the $m$ state. In particular, the $m$ state propagator describes the free-particle evolution in the lattice potential.

The far-field pattern in a direction $\hat{\theta}$ is proportional to the spatial Fourier transform of $d\left(\mathbf{r}, t_{s}\right)$ evaluated at a wavevector $\mathrm{k}$ equal to the projection of the emission wavevector $\hat{\theta} \omega_{1} / c$ along the $x y$ plane. Here, $c$ is the velocity of light. This leads to the following expression for the far-field emission amplitude at a distance $\mathbf{R}=R \hat{\theta}$ :

$$
E_{\hat{\theta}}^{\text {out }}\left(t_{s}\right)=\frac{C_{\mathbf{k}}}{N} \sum_{\mathbf{q}} e^{-i \omega_{m}(\mathbf{q}+\mathbf{k}) t_{s}}\left\langle\phi_{0}\left|\hat{\psi}_{g, \mathbf{q}}^{\dagger}\left(t_{s}\right) \hat{\psi}_{g, \mathbf{q}}(0)\right| \phi_{0}\right\rangle
$$


where $\omega_{m}(\mathbf{q})$ is the free-particle dispersion of $m$ state atoms in the lattice potential and the coefficient $C_{\mathbf{k}}$ is defined as

$$
C_{\mathbf{k}}=\frac{D \omega_{1}^{2} \Omega_{1}(\mathbf{k}) W(\mathbf{k})}{4 \pi \epsilon_{0} R c^{2} \Omega_{2}^{\text {in }}} .
$$

Invariance under translations along the plane guarantees that the coherent emission amplitude in the $\hat{\theta}$ direction, i.e. with an in-plane wavevector $\mathbf{k}$, only depends on the incident probe amplitude $\Omega_{1}(\mathbf{k})$ at the same $\mathbf{k}$. Here we have set $\Omega_{1}(\mathbf{k})=\sum_{j} \Omega_{1}(\mathbf{r}) e^{-i \mathbf{k} \cdot \mathbf{r}_{j}}$, we have defined $\hat{\psi}_{g, \mathbf{q}}=$ $(N)^{-1 / 2} \sum_{j} \hat{\psi}_{g, \mathbf{r}_{j}} e^{-i \mathbf{q} \cdot \mathbf{r}_{j}}$, and we have used a lattice with $N$ sites neglecting boundary effects. The factor $W(\mathbf{k})=$ $\int d^{2} \mathbf{r} w_{g}^{*}(\mathbf{r}) w_{m}(\mathbf{r}) e^{-i \mathbf{k} . \mathbf{r}}$ is a slowly varying envelop stemming from the tight atomic Wannier functions.

Expression (11) relates the coherent amplitude $E_{\hat{\theta}}^{\text {out }}\left(t_{s}\right)$ of the released light to the time-dependent one-body Green function of a generic many-body gas. It is one key result of the present paper. In the limiting case $\omega_{m}(\mathbf{q})=\omega_{m}^{o}$ where the $m$ atoms do not appreciably move during the time $t_{s}$, the far-field amplitude (11) can be further simplified into the form

$$
E_{\hat{\theta}}^{\text {out }}\left(t_{s}\right)=C_{\mathbf{k}} e^{-i \omega_{m}^{o} t_{s}}\left\langle\phi_{0}\left|\hat{\psi}_{g, \mathbf{r}_{i}}^{\dagger}\left(t_{s}\right) \hat{\psi}_{g, \mathbf{r}_{i}}(0)\right| \phi_{0}\right\rangle,
$$

which only involves the local value of the Green function of $g$ atoms.

Experimentally, the coherent $E_{\hat{\theta}}^{\text {out }}$ amplitude can be measured by homodyne detection of the emission with a stronger reference beam at $\omega_{1}$. The intensity and phase of $E_{\hat{\theta}}^{\text {out }}$ is inferred from the amplitude and phase of the oscillations in the interference signal as a function of the mixing phase. This procedure requires coherence at the $g \rightarrow m$ frequency which can be easily achieved if all $\Omega_{1}, \Omega_{2}^{\text {in,out }}$ fields are obtained from a single laser source.

Another quantity of interest is the total (i.e. coherent and incoherent) intensity pattern in either the far- or the near-field. Differently from the coherent amplitude (11), these involve higher order correlations of the many-body gas. For instance, the near-field dipole pattern $I(\mathbf{r})=\left\langle\hat{d}^{\dagger}(\mathbf{r}) \hat{d}(\mathbf{r})\right\rangle$ reads:

$$
\begin{aligned}
I\left(\mathbf{r}, t_{s}\right) & =\frac{|D|^{2}}{\left|\Omega_{2}^{\text {in }}\right|^{2}} \sum_{i, j}\left|\Omega_{1}\left(\mathbf{r}_{j}\right) W_{\mathbf{r}_{i}}(\mathbf{r})\right|^{2} \\
& \times\left\langle\operatorname{vac}\left|\hat{\psi}_{m, \mathbf{r}_{j}}(0) \hat{\psi}_{m, \mathbf{r}_{i}}^{\dagger}\left(t_{s}\right) \hat{\psi}_{m, \mathbf{r}_{i}}\left(t_{s}\right) \hat{\psi}_{m, \mathbf{r}_{j}}^{\dagger}(0)\right| \mathrm{vac}\right\rangle \\
& \times\left\langle\phi_{0}\left|\hat{\psi}_{g, \mathbf{r}_{j}}^{\dagger}(0) \hat{\psi}_{g, \mathbf{r}_{i}}\left(t_{s}\right) \hat{\psi}_{g, \mathbf{r}_{i}}^{\dagger}\left(t_{s}\right) \hat{\psi}_{g, \mathbf{r}_{j}}(0)\right| \phi_{0}\right\rangle .
\end{aligned}
$$

For a localized beam $\Omega_{1}\left(\mathbf{r}_{j}\right)$, the $I(\mathbf{r})$ signal is proportional to a fixed envelope determined by the motion of atoms in the $m$ state times a two-body Green function of $g$ atoms. The correlation function of the $g$ state can be understood as measuring the density at time $t_{s}$ at site $r_{i}$ if one has removed an atom at time 0 at site $r_{j}$. This scheme looks promising e.g. to follow the dynamics of holes in anti-ferromagnetic states [35, 36].

\section{APPLICATION TO BCS SUPERFLUID}

In order to demonstrate the efficiency of the proposed detection technique, we now calculate the signal that is expected for a weakly attractive, unpolarized two-component Fermi gas in an optical lattice at half filling. In particular we show how the proposed method is able to identify a superfluid state and its quasiparticles from the measured two-time correlation function.

In the normal state, the dispersion relation of quasiparticles is given by the free-particle dispersion in the lattice. Here we take the tight-binding form $\hbar \omega_{g, g^{\prime}, m}(\mathbf{q})=\hbar \omega_{g, g^{\prime}, m}^{o}-$ $2 J_{g, g^{\prime}, m}\left[\cos \left(q_{x} a\right)+\cos \left(q_{y} a\right)\right]$. While the $g, g^{\prime}$ atoms feel the same potential, $J_{g}=J_{g^{\prime}}$, the hopping $J_{m}$ for the $m$ state atoms can be different. In the following we set $\omega_{g^{\prime}}^{o}=\omega_{g}^{o}=0$ and focus on the case of half-filling.

In the superfluid state, the quasiparticle dispersion predicted by BCS theory consists of two branches $E_{\mathbf{q}}^{ \pm}=$ $\pm \sqrt{\left[\hbar \omega_{g}(\mathbf{q})\right]^{2}+\Delta^{2}}$ separated by a gap of amplitude $2 \Delta$. The one-body Green function $\mathcal{G}_{g}(\mathbf{q}, t)=\left\langle\hat{\psi}_{g, \mathbf{q}}^{\dagger}(t) \hat{\psi}_{g, \mathbf{q}}(0)\right\rangle$ for the BCS phase reads [34]

$$
\begin{aligned}
\mathcal{G}_{g}(\mathbf{q}, t)=u_{\mathbf{q}}^{2} f\left(E_{\mathbf{q}}^{+}\right) e^{i\left(\omega_{\mathrm{mf}}+E_{\mathbf{q}}^{+} / \hbar\right) t} & \\
& +v_{\mathbf{q}}^{2} f\left(E_{\mathbf{q}}^{-}\right) e^{i\left(\omega_{\mathrm{mf}}+E_{\mathbf{q}}^{-} / \hbar\right) t} .
\end{aligned}
$$

The Bogoliubov coefficients are defined as $u_{\mathbf{q}}^{2}, v_{\mathbf{q}}^{2}=$ $\frac{1}{2}\left[1+\hbar \omega_{g}(\mathbf{q}) / E_{\mathbf{q}}^{ \pm}\right]$and the Fermi distribution as $f(E)=$ $\left(1+e^{E / k_{B} T}\right)^{-1}$. $\omega_{\mathrm{mf}}$ is the mean-field shift [39]. In what follows, we shall focus our attention on low temperature $T$ for which the upper branch $E_{\mathbf{q}}^{+}$is almost empty and can be neglected. Under such an assumption the emission amplitude (11) becomes

$$
E_{\hat{\theta}}^{\text {out }}\left(t_{s}\right)=\frac{C_{\mathbf{k}}}{N} \sum_{\mathbf{q}} e^{-i\left(\omega_{m}(\mathbf{q}+\mathbf{k})-\omega_{\mathrm{mf}}-E_{\mathbf{q}}^{-} / \hbar\right) t_{s}} v_{\mathbf{q}}^{2} f\left(E_{\mathbf{q}}^{-}\right) .
$$

Its Fourier transform with respect to the storage time $t_{s}$ has the form

$E_{\hat{\theta}}^{\text {out }}\left(\omega_{s}\right)=\frac{C_{\mathbf{k}}}{N} \sum_{\mathbf{q}} v_{\mathbf{q}}^{2} f\left(E_{\mathbf{q}}^{-}\right) \delta\left(\omega_{s}-\omega_{m}(\mathbf{q}+\mathbf{k})+\omega_{\mathrm{mf}}+E_{\mathbf{q}}^{-} / \hbar\right)$.

For each value $\omega_{s}$ of the frequency, the signal comes from the wavevectors $\mathbf{q}$ which fulfill

$$
\omega_{s}=\omega_{m}^{o}-\omega_{\mathrm{mf}}+r \omega_{g}(\mathbf{q}+\mathbf{k})-E_{\mathbf{q}}^{-} / \hbar .
$$

In the following we will neglect the contributions by $\omega_{m}^{o}-\omega_{\mathrm{mf}}$ since these can be eliminated in the homodyne detection [40]. Several regimes can be identified depending on the value of the hopping ratio $r=J_{m} / J_{g}$. Experimentally, the hopping amplitudes can be varied within some range by tuning the frequency and polarization of the lattice beams, or, if necessary, by using more complex multi-photon transitions instead than the simple Raman scheme discussed so far.

\section{A. Small hopping ratio $r \ll 1$}

The physics is the simplest in the $r \ll 1$ case where the atoms in the $m$ state do not move during the experiment and 


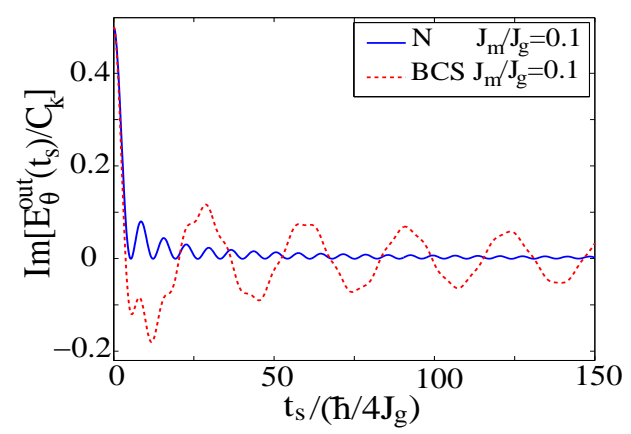

FIG. 3: (Color online) Time-dependence of the emission amplitude in the normal $(\mathbf{k}=0)$ direction for a normal state $\mathrm{N}$ (blue solid line) and a BCS superfluid with gap $\Delta / 4 J_{g}=0.2$ (red dashed line). Hopping ratio $r=0.1$. Temperature $k_{B} T=J_{g} / 50$.

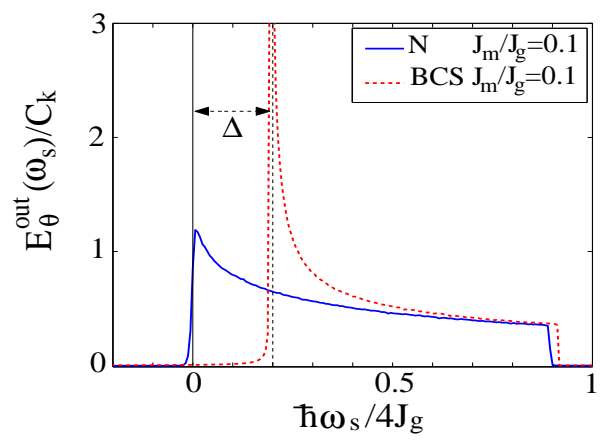

FIG. 4: (Color online) Frequency-dependence of the emission amplitude in the normal $(\mathbf{k}=0)$ direction for a normal state $\mathrm{N}$ (blue solid line) and a BCS superfluid with gap $\Delta / 4 J_{g}=0.2$ (red dashed line). Hopping ratio $r=0.1$. Temperature $k_{B} T=J_{g} / 50$.

the emission amplitude is determined by the local Green function (13). As one can see in Fig. 3, the emission amplitude for a superfluid state as a function of storage time $t_{s}$ shows a slowly decaying oscillation at a low frequency determined by the BCS gap $\Delta$. On top of this slow oscillation, faster and quickly decaying oscillations are visible at frequencies on the order of the Fermi energy (i.e. the band width $J_{g}$ ). The long lasting, slow oscillations are a signature of the superfluid state. They disappear in a normal state where one is left with fast and quickly decaying oscillations.

This physics is easily understood looking at the corresponding frequency spectra plotted in Fig. (4) In the limiting case $r \rightarrow 0$, the spectrum recovers the density of states for quasiparticles. In the normal state, the spectrum has a broad shape extending up to $\hbar \omega_{\max }=4 J_{g}(1-r)$ and showing a singularity at $\omega_{s}=0$ as a consequence of the perfect nesting of the square Fermi surface at half-filling. In the superfluid state, the dominant feature is the peak at $\hbar \omega_{s} \simeq \Delta$ that limits the spectrum from below and from which the BCS gap is immediately extracted. In this state the upper limit of the signal is shifted to $\hbar \omega_{\max }=-4 r J_{g}+\sqrt{16 J_{g}^{2}+\Delta^{2}}$.

\section{B. Equal hopping ratio $r=1$}

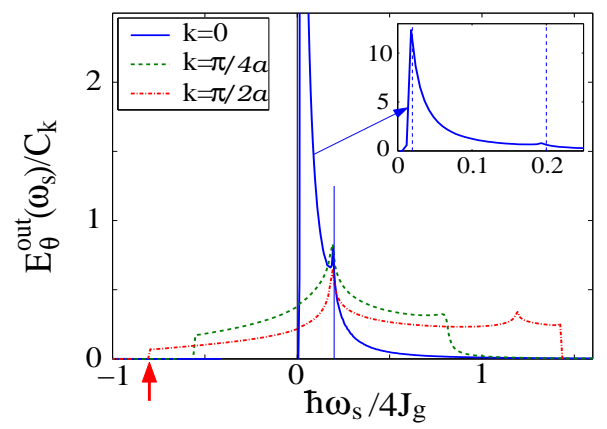

FIG. 5: (Color online) Frequency dependence of the emission amplitude from a BCS superfluid with $\Delta / 4 J_{g}=0.2$ at different angles, $k=k_{x}=k_{y}=0, \pi / 4 a, \pi / 2 a$, with hopping ratio $r=1$. Inset: magnified view of the $\mathbf{k}=0$ curve. The arrows indicate the corresponding spectral minimum $\omega_{\min }$. Temperature $k_{B} T=J_{g} / 50$.

The coherent emission spectra in the case of equal hopping amplitude $r=1$ show a rich structure that strongly depends on the wavevector $\mathbf{k}$ (Fig. 5). Even if the physics is somehow more involved than in the $r \ll 1$ case considered in the previous subsection, still the observed signal can be used to obtain useful information on the many-body system, e.g. its superfluid gap.

Let us first focus on the coherent emission in the $\mathbf{k}=0$ direction. At the lower boundary a large signal is found at $\hbar \omega_{\min } \approx\left(\Delta^{2} / 4 J_{g}\right) / 2$ for $\Delta \ll 4 J_{g}$ (see the inset) which originates from quasiparticles at $\mathbf{q}=0$. The long tail that appears at high frequencies past $\hbar \omega_{s}=\Delta$ is a direct consequence of the smearing out of the Fermi surface on an energy scale $\Delta$ in the BCS state.

The emission spectrum in the direction along the diagonal of the Brillouin zone $\mathbf{k}=(k, k)$ with $k=\pi / 2 a$ ( $a$ is the lattice constant) is characterized by two peaks and a broad background with quite sharp edges: most visible is the strong peak at $\hbar \omega_{s}=\Delta$ that originates from the divergence of the density of states at the Fermi level in a BCS state. This peak persists for different values of $k$ (cf. Fig. $5 k=\pi / 4 a$ ) and its position can be used to experimentally measure the amplitude $\Delta$ of the gap.

\section{High hopping ratio $r \gg 1$}

We conclude our study with a brief account of the case of a high hopping ratio $r \gg 1$. Examples of spectra for $r=3$ are plotted in Fig. 7): in particular, they show a clear peak at $\hbar \omega_{s}=\Delta$ independent of the direction of the light. This distinctive feature allows for a direct measurement of the gap amplitude $\Delta$.

Furthermore, the full dispersion of the BCS quasiparticles $E_{\mathbf{q}}^{-}$can be extracted from the position of the lower edge of the spectrum. For $r \gg 1$ the $r$-dependent term in Eq. (14) dominates and determines the $\mathbf{q}$ values that correspond to 


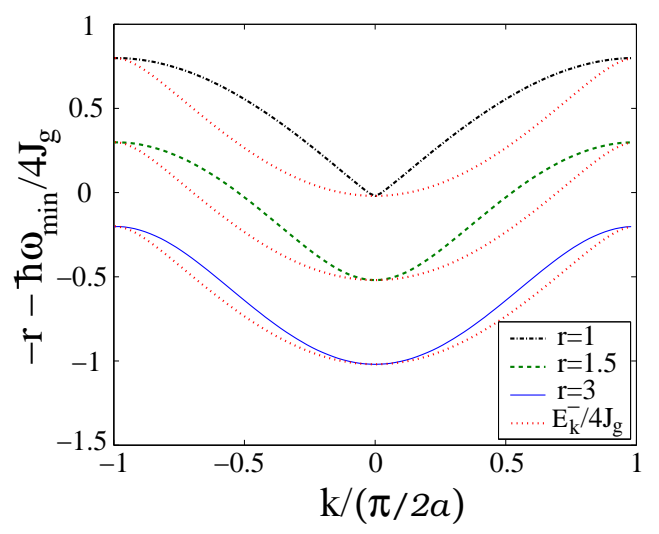

FIG. 6: (Color online) k-dependence of the lower edge of the spectrum compared to the quasiparticle dispersion $E_{\mathrm{k}}^{-}$of the BCS superfluid with $\Delta / 4 J_{g}=0.2$. From top to bottom, hopping ratio $r=1,1.5,3$. Curves for different $r$ are offset by 0.5 for better visibility.

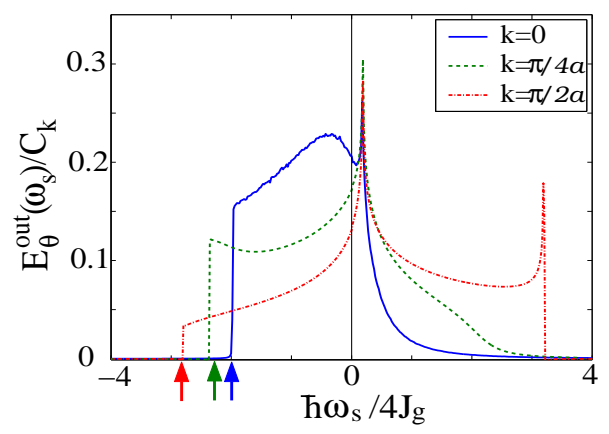

FIG. 7: (Color online) Frequency dependence of the emission amplitude from a BCS superfluid with $\Delta / 4 J_{g}=0.2$ at different angles, $k=k_{x}=k_{y}=0, \pi / 4 a, \pi / 2 a$. Hopping ratio $r=3$. The arrows indicate the corresponding spectral minimum $\omega_{\min }$. Temperature $k_{B} T=J_{g} / 50$.

the spectral edges: the contribution of quasi-particles with momentum $\mathbf{q}=-\mathbf{k}$ determines the sharp lower edge at $\hbar \omega_{\min }=-4 r J_{g}-E_{-\mathbf{k}}^{-}$.

The dependence of the lower spectral edge on the emission direction $\left(k=k_{x}=k_{y}\right)$ is shown in Fig.6for different values of $r$ and compared to the quasi-particle dispersion. While the agreement is limited to the special points $k=0, \pi / 2 a$ for $r=1$, it quickly improves for larger $r$; a reasonably accurate image of the quasi-particle dispersion around $k=0, \pi / 2 a$ is already recovered for $r \gtrsim 3$.

\section{CONCLUSIONS AND PERSPECTIVES}

In summary, we have proposed a novel all-optical, spatially selective and almost non-destructive technique to probe in situ the microscopic many-body dynamics of a gas of interacting ultracold atoms. The technique is inspired to recent light storage experiments and is based on the creation of an atomic coherence by coherent absorption of a pump laser pulse and its later retrieval after a variable storage time: information on the many-body dynamics of the quantum gas is extracted from the amplitude and coherence properties of the retrieved light. Differently from most previous measurement schemes, the use of a spatially localized pump spot will allow to individually address the different coexisting quantum phases that can appear in a trapped system.

The efficiency of the proposed measurement scheme is tested on the specific, analytically tractable example of a twodimensional BCS superfluid. Protocols to extract the superfluid gap and the quasi-particle dispersion are presented, which take into account some most significant difficulties that arise from the internal structure of the atoms.

As our scheme consists of the measurement of two-times correlation functions, it is expected to be of great utility in the study of the non-equilibrium dynamics of a quantum system: on one hand, its almost non-destructive nature suggests that a series of many measurements can be performed at a high repetition rate without significantly perturbing the system dynamics. On the other hand, the observed quantities play a crucial role in the characterization of relaxation dynamics [9, 38]: for instance,they may serve to identify the glassiness of a system in the presence of disorder [9].

Future work will investigate the extension of the method to more complex, three-dimensional geometries: differently from the two-dimensional geometry considered so far, this requires a careful treatment of light propagation across a bulk sample in both the excitation and the retrieval stages. Preliminary work in this direction has appeared as [37].

\section{Acknowledgments}

We are grateful to F. Bariani, M. Capone, M. Inguscio and the Quantum Optics group of ETH Zürich for stimulating discussions. We acknowledge support from the 'Triangle de la Physique', ANR ('FABIOLA' and 'FAMOUS'), the DARPAOLE program, and EPSRC (EP/G029547/1). CK would like to acknowledge the IPAM for its hospitality.
[1] I. Bloch, J. Dalibard, and W. Zwerger, Rev. Mod. Phys. 80, 885 (2008).

[2] M. R. Andrews, M.-O. Mewes, N. J. van Druten, D. S. Durfee, D. M. Kurn, and W. Ketterle, Science 273, 84 (1996).

[3] J. M. Higbie, L. E. Sadler, S. Inouye, A. P. Chikkatur, S. R.
Leslie, K. L. Moore, V. Savalli, and D. M. Stamper-Kurn, Phys. Rev. Lett. 95, 050401 (2005)

[4] M. Köhl, H. Moritz, T. Stöferle, K. Günter, and T. Esslinger, Phys. Rev. Lett. 94, 080403 (2005).

[5] R. Jördens Robert, Niels Strohmaier, Kenneth Günter, Henning 
Moritz, and Tilman Esslinger, Nature 455, 204 (2008).

[6] U. Schneider, L. Hackermller, S. Will, Th. Best, I. Bloch, T. A. Costi, R. W. Helmes, D. Rasch, and A. Rosch, Science 322, 1520 (2008).

[7] L. DeLeo, C. Kollath, A. Georges, M. Ferrero, and O. Parcollet, Phys. Rev. Lett. 101, 210403 (2008).

[8] V. W. Scarola, L. Pollet, J. Oitmaa, and M. Troyer, Phys. Rev. Lett. 102, 135302 (2009).

[9] L. F. Cugliandolo, in Slow relaxation and non-equilibrium dynamics in condensed matter, Les Houches Session 77, J-L Barrat et al eds. (Springer-Verlag, 2003), condmat/0210312.

[10] S. Ritter, A. Öttl, T. Donner, T. Bourdel, M. Köhl, and T. Esslinger, Phys. Rev. Lett. 98, 090402 (2007).

[11] P. Törmä and P. Zoller, Phys. Rev. Lett. 85, 487 (2000).

[12] G.M. Bruun and G. Baym, Phys. Rev. Lett. 93, 150403 (2004).

[13] C. A. Regal and D. S. Jin, Phys. Rev. Lett. 90, 230404 (2003).

[14] S. Gupta, Z. Hadzibabic, M. W. Zwierlein, C. A. Stan, K. Dieckmann, C. H. Schunck, E. G. M. van Kempen, B. J. Verhaar, and W. Ketterle, Science 300, 1723 (2003).

[15] C. Chin, M. Bartenstein, A. Altmeyer, S. Riedl, S. Jochim, J. Hecker Denschlag, and R. Grimm, Science 305, 1128 (2004).

[16] L. Jiang, H. Pu, W. Zhang, and H. Y. Ling, Phys. Rev. A 80, 033606 (2009).

[17] T.L. Dao, A. Georges, J. Dalibard, C. Salomon, and I. Carusotto, Phys. Rev. Lett. 98, 240402 (2007).

[18] J. T. Stewart, J. P. Gaebler, and D. S. Jin, Nature 454, 744 (2008).

[19] C. Kollath, M. Köhl, and T. Giamarchi, Phys. Rev. A 76, 063602 (2007).

[20] C. Liu, Z. Dutton, C. H. Behroozi, and L. V. Hau, Nature, 409, 490, (2001).

[21] U. Schnorrberger, J. D. Thompson, S. Trotzky, R. Pugatch, N. Davidson, S. Kuhr, and I. Bloch, arXiv:0903.0135 (2009).

[22] N. S. Ginsberg, S. R. Garner, and L. V. Hau, Nature 445, 623 (2007).

[23] W. Hofstetter, J.I. Cirac, P. Zoller, E. Demler, and M.D. Lukin,
Phys. Rev. Lett. 89, 220407 (2002).

[24] N. Gemelke, X. Zhang, C.-L. Hung, and C. Chin, Nature 460, 995 (2009).

[25] M. Fleischhauer, A. Imamoglu, and J. P. Marangos, Rev. Mod. Phys. 77, 633 (2005).

[26] Z. Dutton and L. V. Hau, Phys. Rev. A 70, 053831 (2004).

[27] M. Fleischhauer and M. D. Lukin, Phys. Rev. Lett. 84, 5094 (2000).

[28] G. Juzeliunas and P. Öhberg, Phys. Rev. Lett. 93, 033602 (2004)

[29] I. Carusotto, M. Antezza, F. Bariani, S. D. Liberato and C. Ciuti, Phys. Rev. A 77, 063621 (2008)

[30] D.D. Yavuz and N.A. Proite, Phys. Rev. A 76, 041802(R) (2007).

[31] A. V. Gorshkov, L. Jiang, M. Greiner, P. Zoller, and M. D. Lukin, Phys. Rev. Lett. 100, 093005 (2008).

[32] C. H. Schunck, Y. Shin, A. Schirotzek, and W. Ketterle, Nature 454, 739 (2008).

[33] This requires that the timescale of the interaction between $m$ and $g, g^{\prime}$ atoms is much longer than the storage time $t_{s}$.

[34] G. D. Mahan, Many Particle Physics (Plenum, New York, 1981).

[35] R. Strack and D. Vollhardt, Phys. Rev. B 46, 13852 (1992).

[36] G. Sangiovanni, A. Toschi, E. Koch, K. Held, M. Capone, C. Castellani, O. Gunnarsson, S.-K. Mo, J. W. Allen, H.-D. Kim, A. Sekiyama, A. Yamasaki, S. Suga, and P. Metcalf, Phys. Rev. B 73, 205121 (2006).

[37] F. Bariani and I. Carusotto, arXiv:0908.0243 (2009).

[38] P. Calabrese and J. Cardy, J. Stat. Mech.: Theor. Exp. P06008 (2007).

[39] For the attractive Hubbard model with interaction strength $U$ the mean field shift is given by $\hbar \omega_{\mathrm{mf}}=U / 2$.

[40] For the slow outcoupling procedure, the out-coupling laser $\Omega_{2}^{\text {out }}$ can be detuned by the mean-field shift from the resonance $m \rightarrow$ $e$ to eliminate oscillations of frequency $\omega_{m}^{o}-\omega_{\mathrm{mf}}$. 